pattern of combinatorial restraints. The partial cloning and sequencing of several different $b$ alleles ${ }^{7,8}$ did not directly clarify that issue.

Four $b$ alleles have now been sequenced more comprehensively by Gillissen et $a l .^{2}$, and the results are revealing. It turns out that each so-called allele contains two functional genes with unrelated primary sequences but a similar tripartite organization: a constant part, a homeobox domain and a variable region. In memory of the once-divided city, one gene is called $B_{\mathrm{n}}-E$ and the other $B_{\mathrm{n}}-W$. These findings support a new and convincing model for heterodimeric proteins (East and West united), in which all 'homoallelic' dimers have co-evolved for inactivity on account of a particularly fitting configuration, whereas this fit is broken by any formation of heteroallelic dimers. The presence of homeobox domains implies that these DNA-binding proteins have a regulatory function, although the potential target genes have not yet been characterized.

In most cases where mating pheromones and corresponding receptors

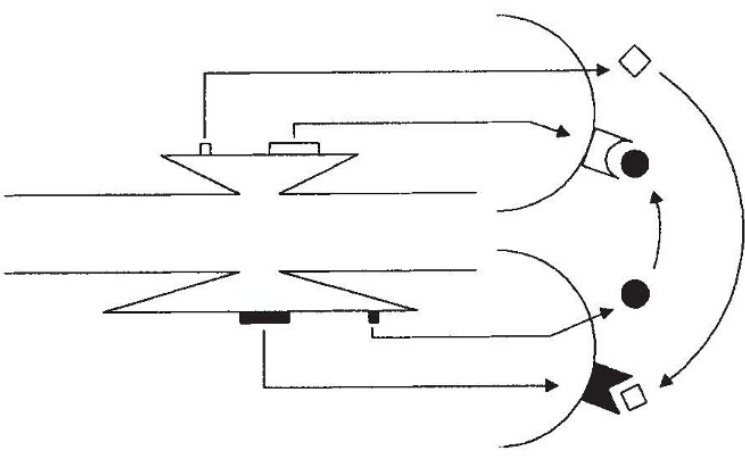

Structure and function of the a locus in Ustilago maydis. The two $a$ alleles resemble insertions, with unrelated sequences of DNA, occupying allelic positions within otherwise homologous chromosomes. The mating-type-specific segments are here termed heterosomal regions. Either one of them carries a short gene for a secreted mating pheromone, as well as a longer gene encoding a membrane-bound receptor which is specific for the opposite pheromone. have been identified, such components only occur in pairs per species. But there are notable exceptions. The ciliate protozoan Euplotes raikovi, for example ${ }^{9}$, has a series of mat alleles, each of which directly controls production of a specific pheromone, and a receptor too. These receptors, however, are not entirely specific for only one pheromone. Rather, they can bind to any pheromone, but they are specifically neutralized by the pheromone of their own mat allele and activated by any other kind. This, at least, is the most convincing explanation of the data, and it is formally analogous to the series of $b$ interactions in Ustilago.

More and more examples of such structural differences at mating-type loci are being identified. Evidently, the ordinary meaning of 'alleles' is blurred when two (or more) functional genes show up at the same locus or map position, and 'idiomorphs' has been suggested as a substitute ${ }^{5,6}$ term. (Originally, the different alleles of ordinary genes were even termed 'allelomorphs', but that designation did not catch on.) On the other hand, alternative clusters of functionally related genes can act as units in their own right, and analogous configurations have occasionally been referred to as 'supergenes'. The driving force seems to be co-evolution of complementary subunits. Because free re-
DAEDALUS

\section{Thick skin}

THE depleted ozone layer is an increasingly uncertain shield against solar ultraviolet light. Daedalus now has a second line of defence. He points out that some dyes, such as acriflavine and gentian violet, stain the skin

permanently. They don't wash off; you have to wait until the skin, in Its slow process of self-renewal from below, sheds the stalned cells from its surface.

So Daedalus is seeking a permanent, colourless but ultraviolet-absorbing skin stain. Some sunscreen preparations use a gallic-acid ester derivative to absorb the ultravlolet. Daedalus recalls those widely distrlbuted natural gallic esters, the vegetable tannins. They bind powerfully to skin proteins - hence the traditlonal tanning process, whlch converts perishable anlmal skin into tough, flexlble leather. Accordingty, DREADCO chemists are devising a tannin derivative which binds firmly to human skin and absorbs ultraviolet IIght. Formulated Into DREADCO's new 'Body Armour' bath salts, It will stain you with a lasting ultraviolet-absorbing layer. The absorption may 'tall' slightly Into the vislble, glving the pleasing Impression of a slight natural tan.

Regular bathing with 'Body Ammour' tannic bath salts will not merely protect you from the ravages of the ultravlolet. It will also tan you like leather, toughening the outer surface of your skin. You will notice nothing. Tough but not brittle, the thin case-hardened surface layer will still fiex easily on the supple tissue beneath. But you will now be armoured against cuts, abraslons, and Insects. Mosquitoes, hornets and wasps will bend their stings vainly against your resistant hlde, while biting insects such as fleas and lice will recoll in bafflement and distaste. For vegetable tannins are one of the plant world's maln defences against insect attack. By blinding to proteins they sabotage an Insect's chemistry; they denature Its masticatory and digestlve enzymes and ruln its appetite.

Body Armour may even protect you from allergles. Many allergles are blamed on the house dust mite. This tiny insect-like creature shares our houses and feeds on our shed skin. Its gut enzyme, spread about in its faocal dust, can cause many distressing allerglc reactions, including dermatitis. (Daedalus reckons this is a cunning evolutionary adaptation to Increase the mites' supply of shed skin). The allergen can be Inactivated by spraying a solution of tannin around the house. But the toughened, tannic skin shed by a Body Armoured household will solve the whole problem. It will denature the enzyme inside the mites, glving them terminal Indigestion.

David Jones 